\title{
Precise Optical Fiber Length Measurement System Based on Fresnel Reflection
}

\author{
Firat Ertac Durak, Arif Basgumus, Serif Ali Sadik and Ahmet Altuncu \\ Electrical \& Electronics Engineering Department, Dumlupinar University, Kutahya 43100, Turkey
}

\begin{abstract}
In this study, a precise optical fiber length measurement system is proposed. The measurement technique is based on the measurement of relative Fresnel reflected light intensity in a test fiber. Time delayed optical reflected pulses are obtained from a single nanosecond pulse injected at the input due to the difference in lengths of the reference and test fibers. The lengths of the different optical fibers have been measured with this technique with high resolution and fast response time. The measured results show that, the proposed technique has a comparable performance with the well-known length measurement systems.
\end{abstract}

Key words: Fresnel reflection, nanosecond pulse, optical fiber length measurement, time delayed pulses.

\section{Introduction}

OTDR (optical time domain reflectometry) has been used for monitoring the fiber optical communication links and measuring the fiber lengths for many years [1]. The principle of OTDR is to measure the Rayleigh backscattered lights and Fresnel reflections as a function of distance [2].

In OTDR, a single short pulse of the light is launched into the fiber and backscattered light is monitored as a function of the distance. The optical power variation in an OTDR trace is affected by different types of imperfections occurred in the test fiber such as fiber attenuation, splice and connector losses and Fresnel reflection from glass-air interface. A theoretical analysis of the OTDR for single mode fibers is presented in Ref. [1]. On the other hand, the OFDR (optical frequency domain reflectometry) technique for detecting very weak reflections in optical fibers is also demonstrated [3]. Eickhoff and Ulrich [4] have proposed coherent OFDR technique for the analysis of attenuation and polarization in moderately long single mode fibers.

The authors in Ref. [5] have presented a new short

Corresponding author: Firat Ertac Durak, Ph.D. student, research fields: fiber optic amplifier and sensor application. length measurement system for fault location in optical waveguide devices based on an interferometric technique using a broadband laser source. The Fresnel reflection based refractive index measurement systems are also demonstrated in Refs. [6-9].

OTDR can measure fiber length using the principles of Rayleigh backscattering. In addition, OTDR can roughly show Fresnel reflections due to large EDZ (event dead zones). In order to measure fiber length in OTDR using Rayleigh backscattering, the output signal pulse width and repetition must be optimized.

In our proposed system, fiber length measurement is only realized using Fresnel reflection. Therefore, the output pulse width of the pulsed laser is only optimized regarding Fresnel reflection and estimated fiber length. In this paper, we demonstrate a fast and precise compact fiber length measurement system based on Fresnel reflection for measuring the lengths of fiber optic cables in site. In the proposed system, a double time-delayed reflection of the optical pulses is generated from a single nanosecond pulse due to the different lengths of the reference and the test fiber. Furthermore, in this design there is no need to use an APD (avalanche photodiode) detector and a long averaging time for measurement since the Fresnel 
reflection intensities are much stronger than Rayleigh backscattering detected and no event dead zone effect. In addition, this measurement system can be manufactured as a lightweight, small and portable handheld device using a proper data acquisition card. The length measurements for different types of SMF (single mode fiber) have shown that our proposed length measurement system is very stable, has fast response time and high resolution.

\section{Experimental Setup}

The basic idea of this technique is to determine the length of the fiber optic cable by measuring differential time between two reflected pulses from the reference fiber and the test fiber ends.

The experimental setup for the proposed system is shown in Fig. 1. A nanosecond optical pulse is generated by a pulsed laser source with a pulse width of about 50 ns and a repetition rate of $10 \mathrm{kHz}$ (Fig. 2) at $1,550 \mathrm{~nm}$ and then it is injected into the first port of the optical circulator. In the proposed system, the factors that determine the minimum and maximum measurement lengths depend on source pulse width and pulse repetition rate. Therefore, the minimum measurement length was around 5 meter due to the need to separate the reflected pulses and the maximum measurement length was around $10 \mathrm{~km}$ due to the need to separate the following periods.

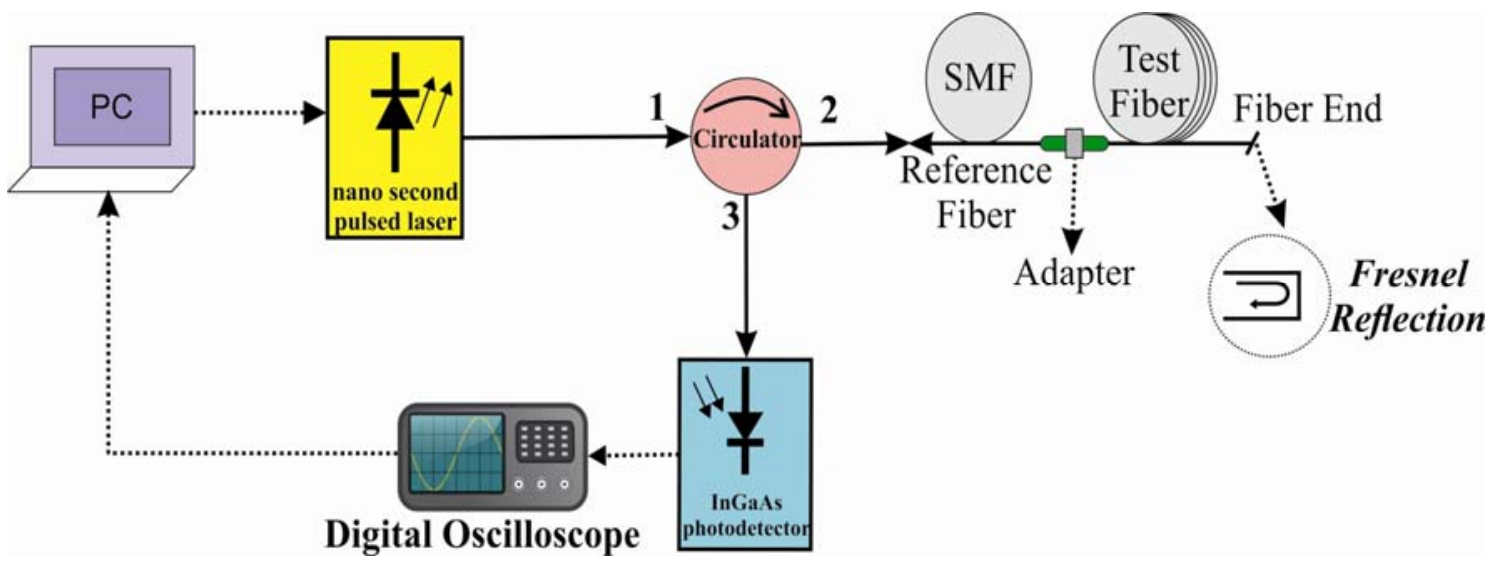

Fig. 1 The experimental setup for the Fresnel reflection-based optical fiber length measurement system.

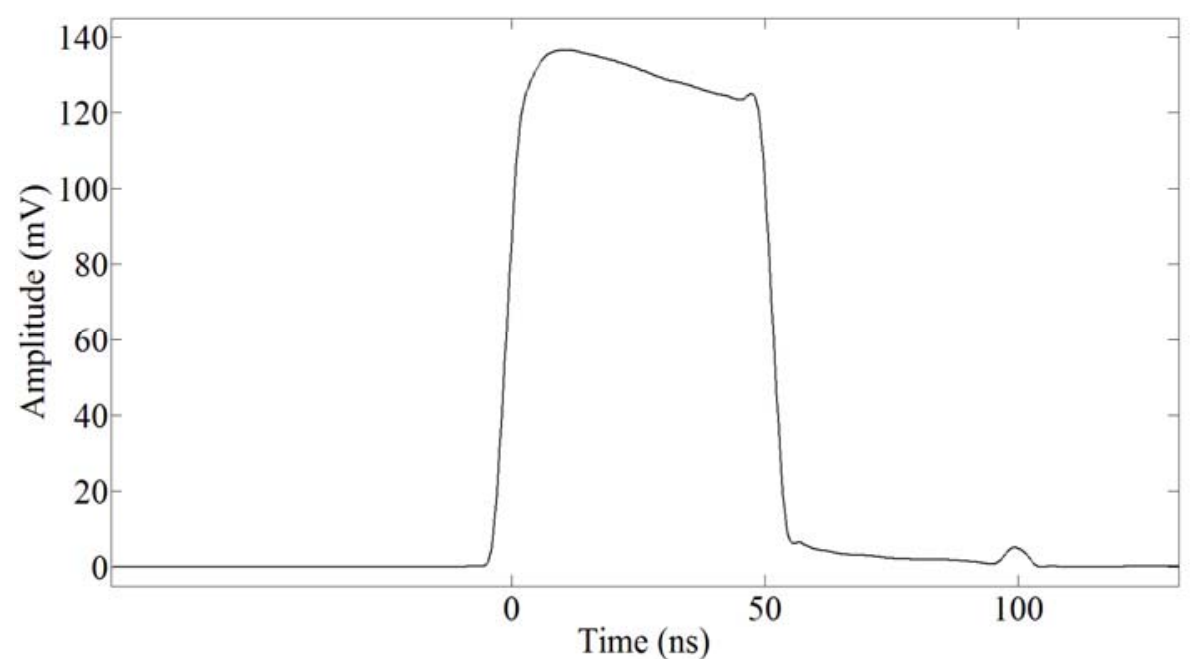

Fig. 250 ns pulses generated by a nanosecond pulsed laser source are launched into the measurement system. The input pulses are partly attenuated to protect the photodetector. 
The second port of the optical circulator is spliced with the reference fiber. The reference and the test fibers are terminated with FC/PC connectors which cause sufficiently strong Fresnel reflections to be able to measure the length of the test fiber. The test fiber is connected to the reference fiber output with an FC/PC adapter. The length information of the test fiber is extracted from the differential time delay for the Fresnel reflected pulses in both ends by a photodetector with $5 \mathrm{GHz}$ bandwidth connected at the third port of the optical circulator. The reflected pulses are measured in the real-time using a $2 \mathrm{GS} / \mathrm{s}$ digital oscilloscope and processed with a computer.

It is accepted that the speed of light is 299,792.458 $\mathrm{km} / \mathrm{s}$ in the vacuum [10]. But, the light travels slower in optical fiber than vacuum because of optical fiber has a refractive index of $n>1$. It is well known that the parameter of refractive index $n$ only defines the refractive index of a core or a cladding layer in a fiber. The value of the effective group index of refraction $n_{\text {eff }}$ is a weighted average for all the indices of the refraction, encountered by the light as it travels within the fiber. Therefore, it represents an actual behavior for the light travelling in a fiber. So, the parameter of $n_{\text {eff }}$ is more useful than the classical refractive index $n$ in the latency applications. The value of $n_{\text {eff }}$ in standard single mode fiber, LEAF (large effective area fiber), NZ-DSF (non-zero dispersion shifted fiber) are 1.4682, 1.4681 and 1.4691 for $1,550 \mathrm{~nm}$, respectively $[11,12]$.

The speed of light in a single mode fiber at 1,550 nm wavelength can be calculated as Ref. [13]

$$
c_{\text {fiber }}=\frac{c}{n_{\text {eff }}}
$$

Using our measurement system, the length of a test fiber ( $L$ ) can be obtained by measuring the time delay of the reflected signals $(\Delta t)$, from the reference and the test fiber ends.

$$
2 L=c_{\text {fiber }} \times \Delta t
$$

Here, the constant 2 means that the light travels two times in the test fiber because of the reflection at the fiber end.

\section{Experimental Results}

Using the proposed measurement system, we have measured the lengths of the different types of fibers with different lengths. In order to improve the accuracy, each measurement was repeated for 25 periods of the reflected pulse. Two reflected pulses are separated in time as shown in Fig. 3.

In our experimental setup, the length of the reference fiber was three meters only so that the reflection time is very close to zero. The differential time delays of the reflected pulses for different test fibers were measured as $\Delta t_{1}=270.5 \mathrm{~ns}$, $\Delta t_{2}=7040 \mathrm{~ns}$ and $\Delta t_{3}=98.1 \mu \mathrm{s}$. The lengths for these fibers could then be calculated from Eqs. (1) and (2) as

$$
\begin{aligned}
& L_{1}=\frac{c_{\text {standard }} \times \Delta t_{1}}{2}=27.617 \mathrm{~m} \\
& L_{2}=\frac{c_{\text {LEAF }} \times \Delta t_{2}}{2}=718.800 \mathrm{~m} \\
& L_{3}=\frac{C_{N Z-D S F} \times \Delta t_{3}}{2}=10009.407 \mathrm{~m} .
\end{aligned}
$$

where the speed of light depends on $n_{\text {eff }}$ in a SMF $\left(c_{\text {fiber }}\right)$ test fiber as in Eq. (1).

It reveals that the proposed system is able to find the almost exact fiber length for the different types of fiber optic cables and linear measurements. 

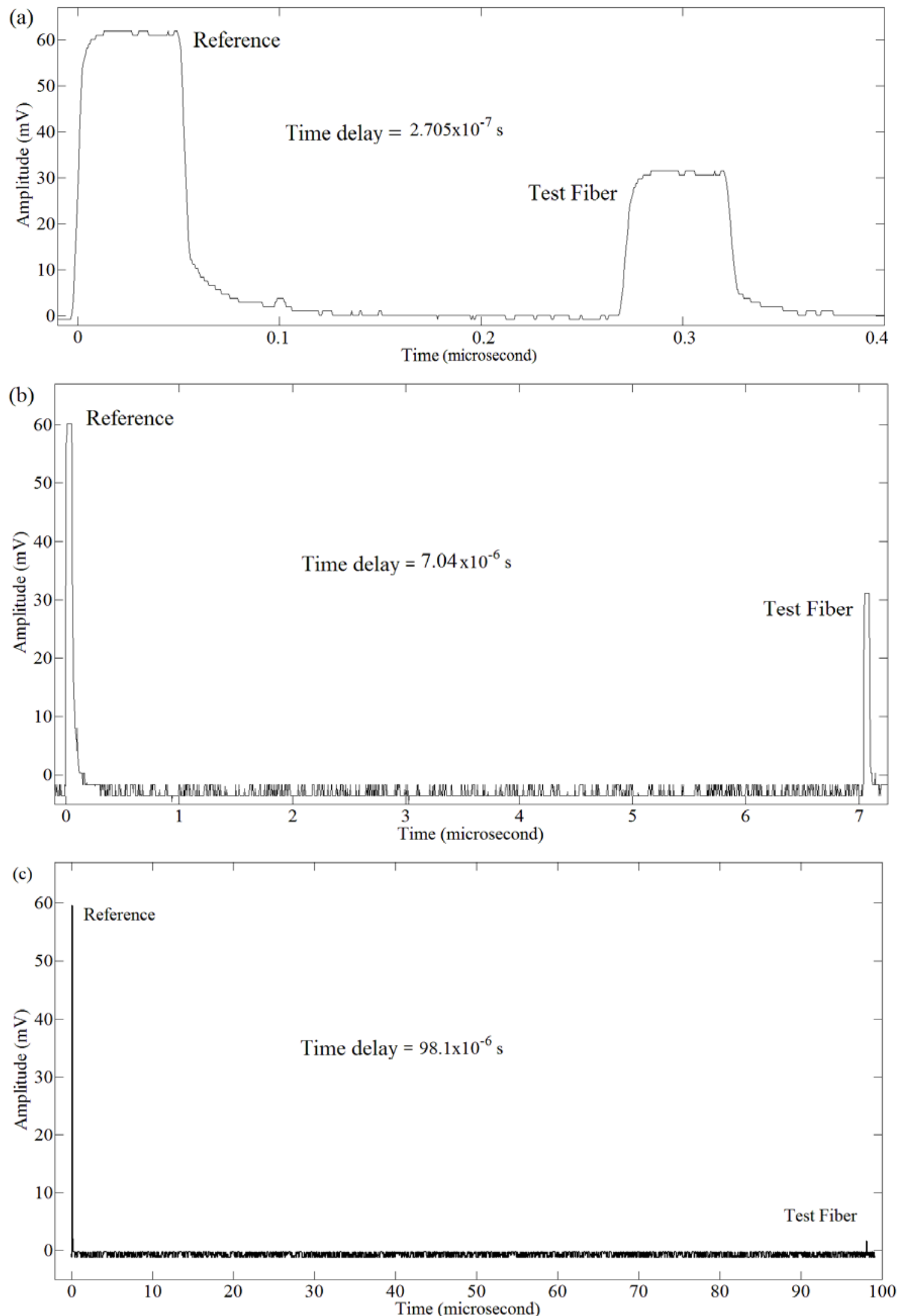

Fig. 3 The time separated reflected pulses from the reference fiber and (a) $27.6 \mathrm{~m}$ standard SMF (b) $719 \mathrm{~m}$ LEAF SMF (c) 10,005 m NZ-DSF. 


\section{Conclusions}

In this paper, we present an ultra-fast and a basic length measurement method for the fiber optic system based on the Fresnel reflection. We measured the time delay of the different optic fiber with high resolution and fast response time. Moreover, we demonstrate that the fiber length can be calculated by the time delay between two reflected signals which are occurring from reference fiber and test fiber ends. Furthermore, the proposed system can measure the length of the broken fiber links which gives a clear Fresnel reflection. However, this system was not considered to precisely measure the position and loss of the macrobends and splices. It should be emphasized that the measurement system is reliable and can be used for the other applications in the fiber optic transmission lines. In addition, this measurement system can be manufactured as a lightweight, small and portable handheld device using a proper data acquisition card.

\section{References}

[1] Philen, D. L., White, I. A., Kuhl, J. F., and Mettler, S. C., 1982. "Single-Mode Fiber OTDR: Experiment and Theory.” IEEE Trans. Micro. Theo. Tech. MTT-30 (10): 1487-96. doi: 10.1109/TMTT.1982.1131282.

[2] Personick, S. D. 1977. "Photon Probe-An Optical-Fiber Time Domain Reflectometer.” The Bell System Technical Journal $56 \quad$ (3): 355-66. doi: 10.1002/j.1538-7305.1977.tb00513.x.

[3] MacDonald, R. I. 1981. "Frequency Domain Optical
Reflectometer.” Applied Optics 16 (10): 1840-4. doi: 10.1364/AO.20.001840.

[4] Eickhoff, W., and Ulrich, R. 1981. "Optical Frequency Domain Reflectometry in Single Mode Fiber.” Appl. Phys. Lett. 39 (9): 693-5. doi: 10.1063/1.92872.

[5] Takada, K., Yokohoma, I., Chida, K., and Noda, J. 1987. "New Measurement System for Fault Location in Optical Waveguide Devices Based on an Interferometric Technique.” Applied Optics 26 (9): 1603-6. doi: 10.1364/AO.26.001603.

[6] Basgumus, A., Durak, F. E., Altuncu, A., and Yilmaz, G. 2016. "A Universal and Stable All-Fiber Refractive Index Sensor System.” IEEE Phot. Tech. Lett. 28 (2): 171-4. doi: 10.1109/LPT.2015.2488040.

[7] Durak, F. E., et al. 2013. "Fresnel Reflection Based Fiber Optic Refractometer.” Journal of Science of Dumlupinar University 32: 13-20.

[8] Giraldi, M. T. M. R., et al. 2014. "Interrogation and Multiplexing System for Fiber Loop Mirror Coupled Intensity Sensors Using OTDR.” Microwave and Optical Tech. Lett. 56 (12): 2860-3. doi: 10.1002/mop.28720.

[9] Basgumus, A., et al. 2014. "Fresnel Reflection Based Fiber Optic Refractive Index Sensor for Liquid Concentration Detection.” IEEE Signal Processing and Comm. App. Conf. (SIU): 594-7. doi: 10.1109/SIU.2014.6830299.

[10] Thyagarajan, K. S., and Ghatak, A. 2007. Fiber Optic Essentials. UK: John Wiley and Sons Ltd., 242.

[11] Corning Inc. 2012. "Explanation of the Sources of Variation in Optical Fiber Effective Group Index of Refraction Values.” White Paper.

[12] Fujikura. 2013. "Specification for Single-Mode Optical Fiber."

[13] Bobrovs, V., Spolitis, S., and Ivanovs, G. 2013. "Latency Causes and Reduction in Optical Metro Networks.” Proc. SPIE 9008. doi: 10.1117/12.2041736. 